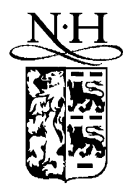

ELSEVIER

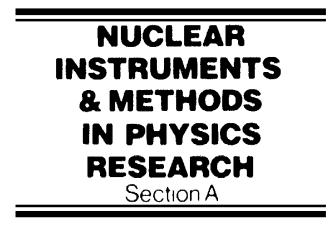

www.elsevier.com/locate/nima

\title{
Extremely compact soft X-ray lasers based on capillary discharges
}

\author{
J.J. Rocca ${ }^{\mathrm{a}, *}$, J. Filevich ${ }^{\mathrm{a}}$, E.C. Hammarsten ${ }^{\mathrm{a}}$, E. Jankowska ${ }^{\mathrm{a}}$, B. Benware ${ }^{\mathrm{a}}$, \\ M.C. Marconi ${ }^{\mathrm{a}}$, B. Luther ${ }^{\mathrm{a}}$, A. Vinogradov ${ }^{\mathrm{b}}$, I. Artiukov, \\ S. Moon ${ }^{c}$, V.N. Shlyaptsev ${ }^{\mathrm{d}}$ \\ ${ }^{a}$ Engineering Research Center, Colorado State University, 1320 Campus Delivery, Fort Collins, CO 80523-1320, USA \\ ${ }^{\mathrm{b}}$ Lebedev Physical Institute, Moscow, Russia \\ ${ }^{\mathrm{c}}$ Lawrence Livermore National Laboratory, Livermore, CA, USA \\ ${ }^{\mathrm{d}}$ University of California at Davis, Livermore, CA, USA
}

\begin{abstract}
Extremely compact high repetition rate soft X-ray lasers based on capillary discharge excitation have demonstrated average powers of a few milliWatt at $46.9 \mathrm{~nm}$, milli-Joule-level pulse energy, peak spectral brightness several orders of magnitude larger than third-generation synchrotron beam lines, and excellent spatial coherence. Examples of the use of a capillary discharge soft X-ray laser in dense plasma diagnostics and laser ablation of materials are summarized.

(C) 2003 Elsevier Science B.V. All rights reserved.
\end{abstract}

PACS: $52.50 . \mathrm{Jm} ; 42.55 . \mathrm{Vc} ; 52.65 . \mathrm{Kj} ; 52.70 . \mathrm{La}$

Keywords: Capillary discharge; Soft X-ray interferometry; Soft X-ray applications

\section{Introduction}

There is significant interest in the development of compact sources of coherent soft X-ray radiation that can be supported by a single regular optical table, yet one capable of producing high (milliWatt) average power. Capillary discharge excitation of Ne-like Ar plasmas has generated such powers of coherent soft $\mathrm{X}$-ray radiation and milli-Joule-level pulses in a table-top setup [1,2]. Multi-Hertz repetition rate operation generated an

\footnotetext{
*Corresponding author. Tel.: +1-970-491-2342; fax: + 1970-491-8671.

E-mail address: rocca@engr.colostate.edu (J.J. Rocca).
}

average power of $\approx 3.5 \mathrm{~mW}$ at a wavelength of $46.9 \mathrm{~nm}$ [2]. The advanced degree of development of this laser is summarized in the next section, followed by a summary of selected experimental results that demonstrate the use of this table-top soft X-ray laser in several diverse areas of science and technology.

\section{Development status of capillary discharge soft $X-$ ray laser}

Large line amplification excited by collisional electron impact excitation of multiply ionized ions has been demonstrated in Ne-like $\operatorname{Ar}(\lambda=46.9 \mathrm{~nm})$ 
[3] Ne-like $\mathrm{Cl}(\lambda=52.9)$ [4] and Ne-like $\mathrm{S}$ $(\lambda=60.8 \mathrm{~nm})$ [5]. The Ne-like Ar capillary discharge laser is the most highly developed of these lasers. Table 1 summarizes the characteristics of this laser and its present output parameters. These laser output parameters were obtained utilizing aluminum oxide capillary channels $3.2 \mathrm{~mm}$ in diameter filled with preionized Ar gas at an optimized pressure of $\approx 460 \mathrm{mT}$ orr. The plasma columns were excited by current pulses of $\approx 26 \mathrm{kA}$ peak amplitude with a $10-90 \%$ rise time of approximately $40 \mathrm{~ns}$. Fig. 1 illustrates the size of the capillary discharge Ne-like Ar laser. This capillary discharge-pumped laser occupies a table space of approximately $0.4 \mathrm{~m} \times 1 \mathrm{~m}$, a size comparable to that of many widely utilized visible or ultraviolet gas lasers.

In this laser, the excitation current pulse is produced by discharging a water capacitor

Table 1

Summary of the parameters for the $46.9 \mathrm{~nm}$ capillary discharge table-top soft X-ray laser

\begin{tabular}{lll}
\hline Laser parameter & & Ref. \\
\hline Pulse energy & $0.88 \mathrm{~mJ}$ at $4 \mathrm{~Hz}$ & {$[2]$} \\
Average power & $3.5 \mathrm{~mW}$ & {$[2]$} \\
Peak power & $0.6 \mathrm{MW}$ & {$[2]$} \\
Divergence & $\approx 4.6 \mathrm{mrad}$ & {$[2,3]$} \\
Pulse width & $1.2-1.5 \mathrm{~ns}$ & {$[2,3]$} \\
Peak spectral brightness & $2 \times 10^{25}$ photons/ & {$[6]$} \\
& $\left(\mathrm{s} \mathrm{mm}^{2} \mathrm{mrad}^{2}\right.$ & \\
& $0.01 \%$ bandwidth $)$ \\
\hline
\end{tabular}

through a spark gap switch connected in series with the capillary load. The laser average output pulse energy was measured to increase from $0.075 \mathrm{~mJ}$ for a plasma column $16 \mathrm{~cm}$ in length, to $0.88 \mathrm{~mJ}$ for the plasma column $34.5 \mathrm{~cm}$ in length. At the output of the longest capillary the laser beam intensity approaches $1 \mathrm{GW} / \mathrm{cm}^{2}$, and exceeds the saturation intensity by more than an order of magnitude. Fig. 2a shows the shot-to-shot variations of the measured laser output pulse energy and corresponding laser average power for a $34.5 \mathrm{~cm}$ long discharge operated at $4 \mathrm{~Hz}$ repetition rate. The average laser output power is about $3.5 \mathrm{~mW}$, corresponding to $>8 \times 10^{14}$ photons $/ \mathrm{s}$ [2]. Fig. $2 \mathrm{~b}$ shows the average laser output energy per pulse is $0.88 \mathrm{~mJ}$ and that the energy of the highest energy pulses exceeds $1 \mathrm{~mJ}$. More than 5000 laser shots were obtained from a single capillary. The full-width at half-maximum (FWHM) of the corresponding laser pulse is $1.5 \pm 0.05 \mathrm{~ns}$, longer than the $1.2 \mathrm{~ns}$ pulsewidth that we measured for an $18.2 \mathrm{~cm}$ long amplifier [3]. The average peak laser output power obtained with the longest plasma column is $\approx 0.6 \mathrm{MW}$. The output beam intensity distribution has an annular shape. The peak-to-peak divergence was measured to be about $4.6 \mathrm{mrad}$ for all capillary lengths between 18 and $34.5 \mathrm{~cm}$. Recent measurements of the spatial coherence indicate that full spatial coherence is approached with the longest capillaries and that the peak spectral brightness is about $2 \times 10^{25}$ photons $/\left(\mathrm{s} \mathrm{mm}^{2} \mathrm{mrad}^{2} 0.01 \%\right.$

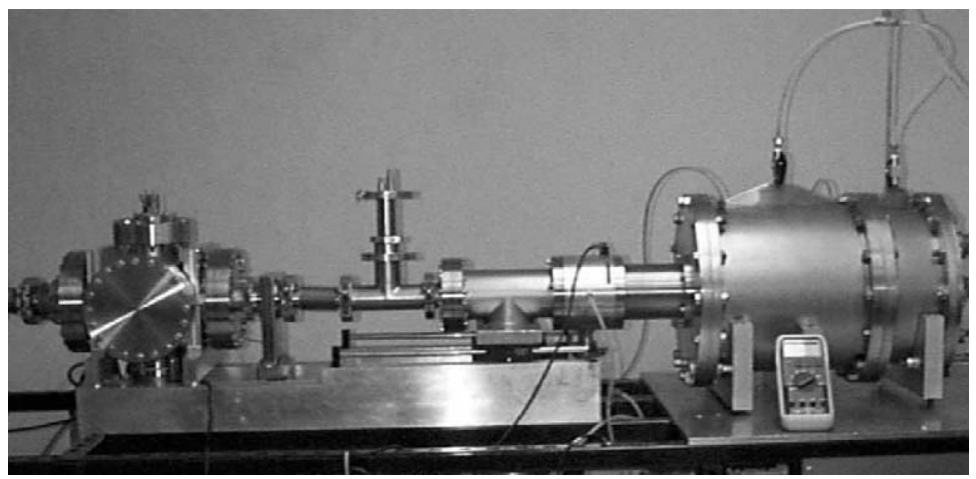

Fig. 1. Photograph of capillary discharge soft X-ray laser (right) and applications chamber (left). A multimeter is shown in front of the laser to provide size reference. 

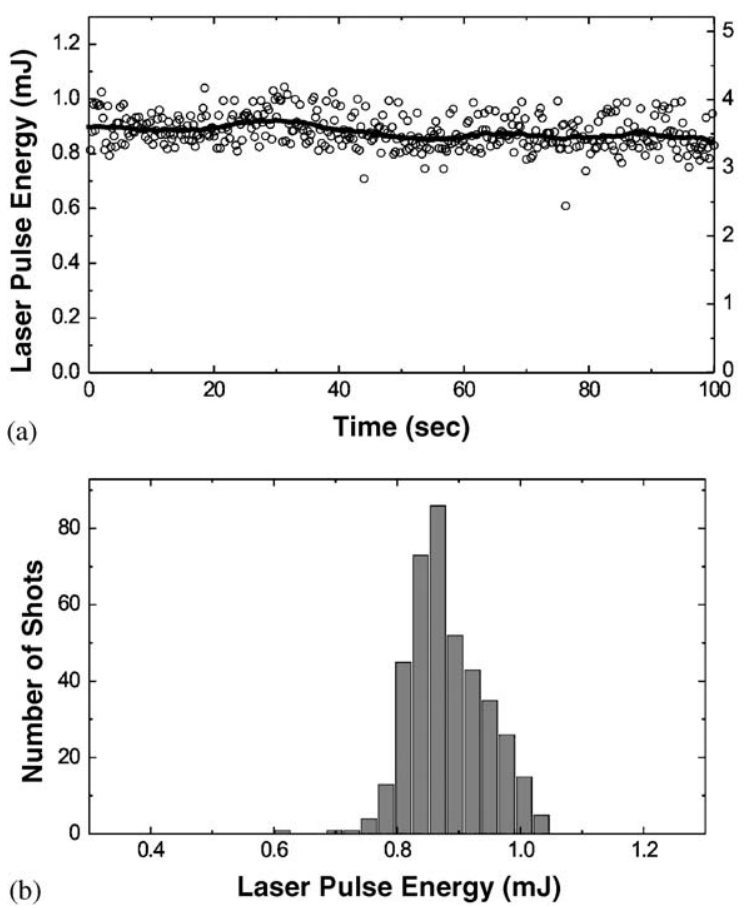

Fig. 2. Measured output pulse energy and average power of a table-top capillary discharge $46.9 \mathrm{~nm}$ laser operating at a repetition frequency of $4 \mathrm{~Hz}$ : (a) Shot-to shot laser output energy and average output power computed as a walking average of 60 continuous laser pulses; and (b) distribution of the output pulse energy. Average pulse energy is $0.88 \mathrm{~mJ}$ and the standard deviation is $0.06 \mathrm{~mJ}$.

bandwidth) [6]. The possibility of extending these capillary discharge laser sources to shorter wavelengths is currently under investigation [7].

\section{Applications}

Below, we discuss the use of the $46.9 \mathrm{~nm}$ laser in dense plasma interferometry and material ablation. The application of this laser to plasma shadowgraphy [8] and soft X-ray reflectometry for the determination of optical constants [9] has been discussed in recent publications.

\subsection{Soft X-ray laser interferometry of dense plasmas}

The Soft X-ray lasers allow the probing of largescale high-density plasmas beyond the limits that plasma refraction and absorption impose to visible and ultraviolet laser probes. Da Silva et al. conducted the first soft X-ray laser interferometry experiments using a laboratory-size $15.5 \mathrm{~nm} \mathrm{Ne}$ like $\mathrm{Y}$ laser pumped by the Nova laser in conjunction with a Mach-Zehnder interferometer based on thin-film beam splitters [10]. Capillary discharge-pumped soft X-ray lasers, with an extremely high brightness that is similar or higher than that of their laboratory-size predecessors and a much higher repetition rate, offer the opportunity to develop portable soft X-ray tools for the diagnostics of a large variety of dense plasmas. Our group has realized the first demonstrations of soft X-ray interferometry using a table-top laser [11-13]. Experiments with the capillary discharge $46.9 \mathrm{~nm}$ Ne-like Ar laser were conducted using either a wavefront-division interferometer based on Lloyd's mirror [11,12], or an amplitude division interferometer in which diffraction gratings were used as beam splitters [13].

The soft X-ray laser interferometry system used in our most recent experiments is schematically shown in Fig. 3. It consists of a table-top capillary discharge laser emitting $\sim 0.1 \mathrm{~mJ}$ pulses of $\sim 1.2 \mathrm{~ns}$ duration at $46.9 \mathrm{~nm}$, the diffraction grating interferometer (DGI), and a Nd:YAG laser that is focused onto a polished $\mathrm{Cu}$ target to generate the plasmas that are the subject of this study.

The DGI consists of a modified Mach-Zehnder configuration of rhomboidal shape that uses diffraction gratings as beam splitters and elongated grazing incidence mirrors. The zero and first diffracted orders from the first grating are used to form the two arms of the interferometer. The elongated gold-coated mirrors redirect the beam towards the second grating where they are recombined and the interference pattern is generated. Advantages of the DGI scheme over other amplitude-division soft X-ray interferometers based on thin-film beam splitters $[10,14]$ include a higher throughput ( $\sim 6 \%$ percent per arm) and a significantly increased resistance of the beam splitters to plasma debris. A $\mathrm{Si} / \mathrm{Sc}$ multilayers optics system was used to image the plasma onto a $\mathrm{CCD} / \mathrm{MCP}$ detector setup with $51.2 \times$ magnification. The alignment of the interferometer was done with an $824 \mathrm{~nm}$ laser diode on a second set of 


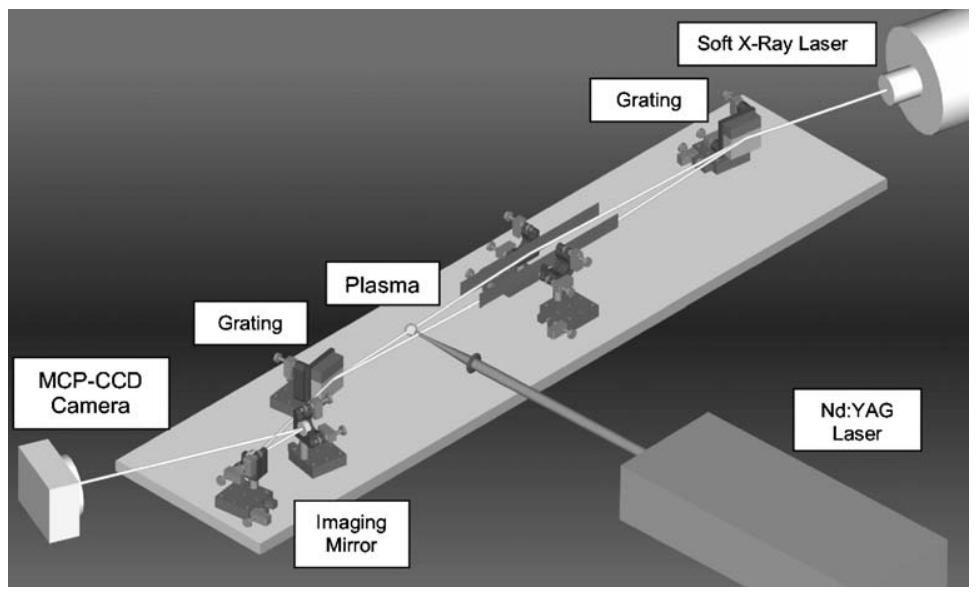

Fig. 3. Schematic representation of the experimental setup. The interferometer was at $\sim 2 \mathrm{~m}$ from the exit of the soft X-ray laser. The detector was placed at $\sim 7 \mathrm{~m}$ from the interferometer.

rulings on the gratings. In the elongated plasma columns generated by the capillary discharge, gain guiding and strong refractive anti-guiding results in the rapid buildup of spatial coherence [6]. This intrinsic mode selection mechanism makes it possible to achieve a very high degree of spatial coherence, which in turn allows for the generation of high-visibility interferograms. The interferograms obtained have a fringe visibility of $\sim 0.5$ over the entire $400 \times 500 \mu \mathrm{m}^{2}$ field of view. Below, we exemplify the use of this interferometry setup in the characterization of a spot-focus laser-created plasma.

In this experiment, the plasmas were formed from a copper target using $0.62 \mathrm{~J}$ pulses of $13 \mathrm{~ns}$ FWHM duration from an Nd:YAG laser operating at its fundamental wavelength of $1.06 \mu \mathrm{m}$. The laser was focused onto a $\sim 30 \mu \mathrm{m}$ diameter spot using a $15 \mathrm{~cm}$ focal length aspheric lens. Detailed series of interferograms were obtained for plasmas created by firing the heating laser either onto a fresh polished copper target or into the crater formed by previous shots. In the latter case, the plasmas display a significant density increase at large distances from the target surface. This results from the fact that the crater constrains the plasma's lateral expansion and guides its motion into the direction normal to the target. In all cases, the measurements unveiled the formation of an "inverted center" electron density profile with a density minimum on axis, as we also observed in the line-focus experiments at lower irradiation intensities [15]. The plasma evolution was mapped by taking interferograms at different times relative to the initiation of the heating laser pulse, shown in Fig. 4.

A concave shape is visible in the fringes throughout the plasma evolution. In this axisymmetric geometry such fringe pattern is indicative of a concave electron density profile with a significant minimum on axis. At the time of the maximum laser intensity, this central minimum in the density profile is observed to extend through practically the entire sub-critical region of the plasma. Fig. 5 shows the evolution of the electron density distributions derived from Abel inversion of the interferograms of Fig. 4. The formation of a concave electron density profile with pronounced plasma sidelobes and a density cavity on the irradiation axis is observed. At $10.5 \mathrm{~ns}$ (not shown), the electron density of the sidelobes is measured to reach $\sim 9 \times 10^{20} \mathrm{~cm}^{-3}$ at a distance of $27 \mu \mathrm{m}$ from the target. This density corresponds to $90 \%$ of the critical density of this $\lambda=1.06 \mu \mathrm{m}$ pump laser.

This observed "inverted" density profile is neither a result of the ponderomotive force nor the effect of laser radiation refraction or filamentation. Hydrodynamic simulations show that the observed two-dimensional profiles are caused by 

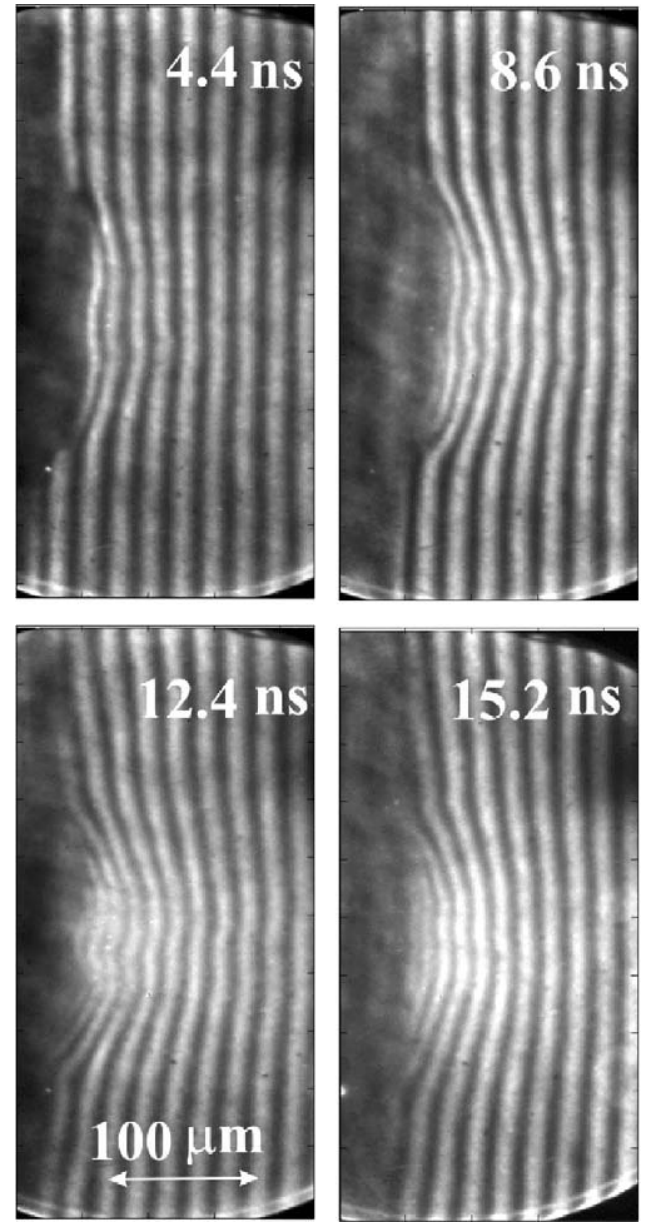

Fig. 4. Interferograms of plasmas generated focusing an NdYAG laser into a spot $\sim 30 \mu \mathrm{m}$, with an irradiation intensity of $7 \times 10^{12} \mathrm{~W} \mathrm{~cm}^{-2}$. The interferograms correspond to plasmas generated firing the laser for a second time in the same target location. The times delays are measured with respect to the beginning of the laser pulse. The heating laser is incident from the right.

the generation of cold plasma sidelobes outside the laser-irradiated target area and by the subsequent establishment of pressure balance between the sidelobes and the hotter central plasma. The sidelobes result from the buildup of cold material generated by an increased ablated area caused mainly by XUV plasma radiation. Moreover, the simulations indicated that this is essentially a universal effect that should be observed over a wide range of plasma parameters. Similar results for line-focus plasmas are discussed in detail in another paper [15].

\subsection{Soft X-ray laser ablation}

Focusing of these laser beams with multilayercoated optics has potential to reach very high intensities and energy densities, opening new applications for short-wavelength coherent radiation. Here, we summarize the characterization of a focused $46.9 \mathrm{~nm}$ laser beam generated by a Ne-like Ar capillary discharge soft X-ray laser, and the results of the first laser ablation experiment with a soft X-ray laser [16].

The soft X-ray laser pulses had an energy of about $0.13 \mathrm{~mJ}$ and $1.2 \mathrm{~ns}$ FWHM duration and were generated at a repetition rate of $1 \mathrm{~Hz}$ by amplification in an $18.2 \mathrm{~cm}$ long $\mathrm{Ar}$ capillary plasma. The laser beam was focused by a spherical $(R=10 \mathrm{~cm}) \quad \mathrm{Si} / \mathrm{Sc}$ multilayer-coated mirror of $\approx 40 \%$ normal incidence reflectivity located in a vacuum chamber at $256 \mathrm{~cm}$ from the exit of the capillary amplifier. The focused laser beam was observed to have sufficient intensity to ablate aluminum, stainless steel and brass when the samples were positioned within several hundred micrometers of the focus. Series of imprints of the beam for positions for different targets along the optical axis were obtained by continuously moving the translation stage while repetitively firing the laser at a repetition rate of $1 \mathrm{~Hz}$.

Fig. 6 is a scanning electron microscope (SEM) photograph of the surface of a brass target showing the progression of ablation patterns obtained as the target was moved away from the mirror and towards the focus. Each ablation pattern is the result of a single laser shot. At an axial distance of a few hundred micrometers from the focal region, the ablation patterns have the shape of thin annular disks. As the focal region is approached, the thickness of the ablated rings increases and a central spot develops. The depth of the rings was measured to be $\approx 2 \mu \mathrm{m}$. Finally, very near the focus, the patterns evolve into a single spot with a deep hole on axis. The smallest spot has an outer diameter of about $17 \mu \mathrm{m}$ and contains a deep central hole of about $2-3 \mu \mathrm{m}$ diameter. 


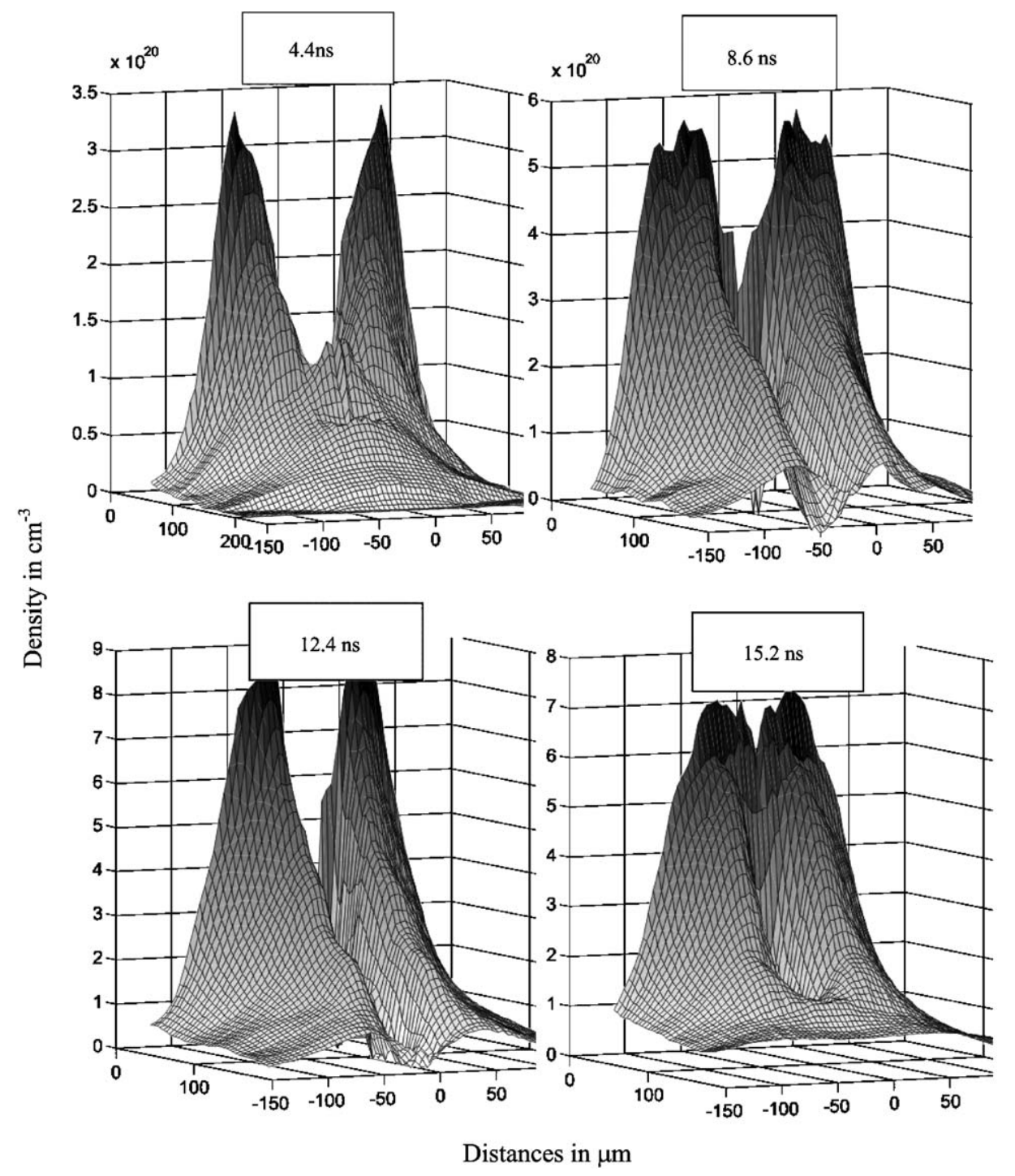

Fig. 5. Computed electron density corresponding to the interferograms of Fig. 4.

Fig. 7 shows the radial cross-section of the beam intensity distributions in the focal region. For comparison with the experiment, the measured boundaries of the ablated regions are represented as black dots in the same figure. All the major features of the observed ablation profiles of Fig. 6 are well described by ray tracing computations. These features are the result of the spherical aberration that causes the rays to converge and cross at those locations. Similarly, the spherical aberration causes the central peak, which begins to develop when the outermost rays converge on the axis. Near the so-called "plane of minimum confusion" the intensity distribution is dominated by the sharp central peak, which is responsible for the deep central hole observed in the SEM images. The average intensity within a $2 \mu \mathrm{m}$ diameter region is estimated to be greater than $1 \times 10^{11} \mathrm{~W} / \mathrm{cm}^{2}$ from the computed fraction of rays that intersect this region. Recent wavefront measurements indicate 

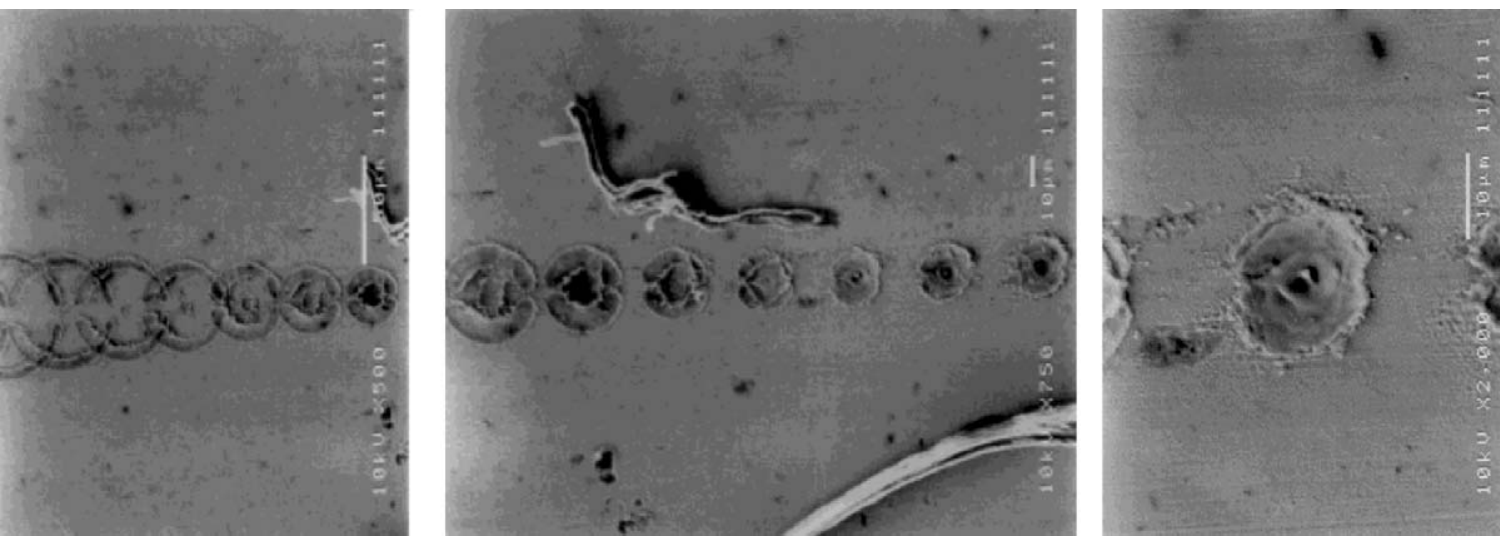

Fig. 6. SEM images of the ablation patterns obtained by moving a brass sample towards the focus. Each ablation pattern corresponds to a single laser shot. The composition of the brass used in this experiment was by weight $60.95 \% \mathrm{Cu}, 39.05 \% \mathrm{Zn} \mathrm{with} \mathrm{small} \mathrm{Al}$ impurities.

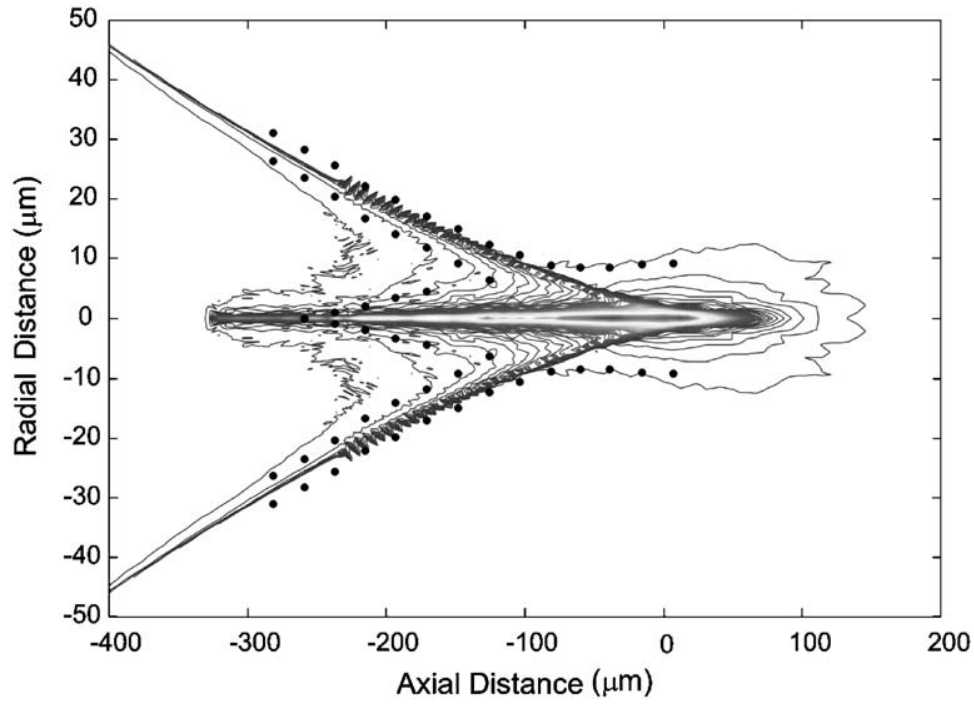

Fig. 7. Computed radial cross-section of the beam in the focal region. The dots correspond to the measured boundaries of the ablated patterns.

that the use of aspheric optics should allow focusing to intensities $>10^{13} \mathrm{~W} / \mathrm{cm}^{2}$ [17].

\section{Conclusions}

In summary, very compact capillary dischargepumped table-top soft X-ray lasers have reached a level of development that allows their routine use in numerous applications. The average coherent power per unit of spectral bandwidth of the 46.9 Ne-like Ar laser is similar to that of a thirdgeneration synchrotron beam line, and its highpeak spectral brightness makes it one of the brightest sources of soft X-ray radiation in this spectral region. 


\section{Acknowledgements}

The development of the soft X-ray laser was supported by the National Science Foundation. The plasma interferometry work was supported by the US Department of Energy Grant DE-FG0302NA00062. We gratefully acknowledge a grant from the W.M. Keck Foundation, and the US Civilian Research and Development Foundation (CRDF).

\section{References}

[1] J.J. Rocca, Rev. Sci. Instrum. 70 (1999) 3799.

[2] C.D. Macchietto, B.R. Benware, J.J. Rocca, Opt. Lett. 24 (1999) 1115.

[3] B.R. Benware, C.D. Macchietto, C.H. Moreno, J.J. Rocca, Phys. Rev. Lett. 81 (1998) 5804.
[4] M. Frati, M. Seminario, J.J. Rocca, Opt. Lett. 25 (2000) 1022.

[5] F.G. Tomasel, J.J. Rocca, V.N. Shlyaptsev, C.D. Macchietto, Phys. Rev. A 55 (1997) 1437.

[6] Y. Liu, et al., Phys. Rev. A 63 (2001) 033802.

[7] S. Sakadzik, et al., Proceedings on "Soft X-ray Lasers and Applications IV", SPIE J., Vol 4505, San Diego, 1-3 August 2001.

[8] M.C. Marconi, et al., Phys. Rev. E 62 (2000) 7209.

[9] I.A. Artioukov, et al., IEEE J. Sel. Topics Quantum Electron. 5 (1999) 1495.

[10] L.B. Da Silva, et al., Phys. Rev. Lett. 74 (20) (1995) 3991.

[11] C.H. Moreno, et al., Phys. Rev E. 60 (1) (1999) 911.

[12] J.J. Rocca, C.H. Moreno, M.C. Marconi, K. Kanizay, Opt. Lett. 24 (1999) 420.

[13] J. Filevich, et al., Opt. Lett. 25 (2000) 356.

[14] A.S. Wan, et al., Phys. Rev. E 55 (1997) 6293.

[15] J. Filevich, et al., Proceedings of the Eighth International Conference on X-ray Lasers, Aspen, CO, USA, May 2002.

[16] B.R. Benware, et al., Opt. Lett. 24 (1999) 1724.

[17] S. Le Pape, et al., Phys. Rev. Lett. 88 (2002) 183901. 